\title{
Analysis of processing time for conjunctive and disjunctive problem solving*
}

\author{
NANCY WADSWORTH DENNEY \\ University of Washington, Seattle, Wash. 98105
}

A comparison of latencies and S-paced postfeedback intervals (PFIs) on positive- and negative-instance trials indicated that neither PFI nor latency varied as a function of the type of instance. However, a comparison of PFIs and latencies on correct and incorrect response trials indicated that both PFIs and latencies were longer on error trials than on correct response trials. Thus, it was concluded that more information processing occurred on error trials. Instructions to focus the $\mathrm{Ss}$ on positive, negative, or positive and negative instances had negligible effects on performance.

Recent comparisons of different percentages of positive and negative instances in stimulus presentation can be interpreted as indicating that the type of instance from which the $S$ gets most information depends on the particular rule with which he is working. Bourne \& Guy (1968) compared performance on conjunctive, disjunctive, and conditional problems after training on all positive, all negative, or a combination of positive and negative instances. They found that under the attribute-identification paradigm, in which Ss were told the rule and required to learn the attributes which were combined by that rule, the Ss generally performed best when trained with instances from the smaller, more homogeneous class. The opposite was obtained when the Ss were given the two relevant attributes and required to learn the rule by which the attributes were combined. In her complete learning situation, in which Ss were required to learn both rule and relevant attributes, Denney (1969) found that conjunctive and disjunctive problems were solved faster when the relative concentration of instances from the smaller class-the positive class on the conjunctive problem and the negative class on the disjunctive problem was increased. One aim in conducting the present study was to investigate further the Ss' use of positive and negative instances in conjunctive and disjunctive problems by comparing the lengths of S-paced postfeedback intervals (PFIs) after positive and negative instances.

A second aim was to determine if performance could be either facilitated or impaired with instructions to attend more to either positive or negative instances and

* This research was supported by a National Institute of Mental Health research fellowship, $\mathrm{MH}$ 34,313-01A1, from the United States Public Health Service. The author wishes to express her appreciation to Dr. Joseph C. Campione for his advice with respect to various aspects of the study and to Douglas R. Denney for his help with the data analysis. also if instructions to attend more to a particular type of instance would have any effect on the length of the PFIs. In order to explain the relative difficulty of the disjunctive rule in relation to the conjunctive rule, it has been hypothesized that Ss learn to focus on the positive class sooner than they learn to focus on the negative class (Conant \& Trabasso, 1964; Denney, 1969). Thus, the conjunctive problem should be solved faster because the Ss learn to focus on the most useful class (the smallest class) first, while on the disjunctive problem the Ss learn to focus on the least useful class (the largest, more heterogeneous class) first. If this is the case, then instructions that focus the $S$ on the positive class should facilitate conjunctive problem solution and instructions that focus the $S$ on the negative class should facilitate disjunctive problem solution because these instructions would be focusing the $S$ on the more useful class. Likewise, instructions that focus the $S$ on the positive class should hinder disjunctive problem solution and instructions that focus the $S$ on the negative class should hinder conjunctive problem sulution. In order to test these hypotheses, four sets of instructions were employed: (1) Control Instructions 1 in which no mention was made of either positive or negative instances; (2) Control Instructions 2 in which the Ss were told to pay attention to both positive and negative instances; (3) instructions in which the Ss were told to pay special attention to positive instances; and (4) instructions in which the Ss were told to pay special attention to negative instances.

A third aim was to attempt to replicate the finding of Wells \& Laestadius (1968) that S-paced PFIs are longer after error trials $(4.4 \mathrm{sec})$ than after correct response trials $(3.3 \mathrm{sec})$. Although this finding is predicted by Levine (1966), it is somewhat surprising in light of the results of two E-controlled PFI studies. In their E-controlled condition, Wells and
Laestadius found that performance was better in the condition in which PFIs were long after correct responses than in the condition in which PFIs were long after incorrect responses. Bourne, Dodd, Guy, \& Justesen (1968), on the other hand, found that any E-controlled increase in PFI facilitated performance, regardless of whether the increase followed a correct or incorrect response. In addition to PFIs, latencies were also recorded for exploratory purposes.

\section{SUBJECTS}

Eighty introductory psychology students from the University of Washington were assigned randomly to the eight experimental groups.

$$
\text { STIMULI }
$$

The stimulus slides consisted of the 16 possible combinations of the following four binary dimensions: (1) color (red-green); (2) form (square-triangle); (3) size (6-12 in.); and (4) pattern on figure (stripes-dots). The stimulus patterns were randomized with the restrictions that there were an equal number of positive and negative instances and that no response category occurred more than three times consecutively.

\section{EXPERIMENTAL DESIGN}

The experimental design was a mixed design with two between-Ss variables-rule (conjunctive-disjunctive) and instructions (Control 1, Control 2, positive focusing, and negative focusing)-and three within-Ss variables-type of instance (positive-negative), type of response (correct-incorrect), and trial block (first-second). For each $S$ the trials prior to criterion were divided in half; the first half of the trials were Trial Block 1 and the second half were Trial Block 2. PROCEDURE

Each $S$ was brought individually into the experimental room and was seated facing the wall onto which the slides were projected. The E, seated directly behind the $\mathrm{S}$, gave one of four sets of instructions depending on the experimental condition to which the $\mathrm{S}$ was assigned. The Control 1 instructions were as follows: "This is a concept-learning experiment. Your task will be to learn the concept. You will be shown a series of geometrical figures one at a time on the wall in front of you. Some of these figures will be examples of the concept and some will not be examples of the concept. When a figure comes on, tell me whether you think it is or is not an example of the concept and then I will tell you whether you are right or wrong. When you are ready to go on to the next pattern. just press this button and the next slide will come on. This procedure will continue until you get 16 right in a row." In each of the other instruction conditions, 
Table 1

Results (Fs) of All PFI and Latency Comparisons Made on Combined Instruction Conditions

\begin{tabular}{lcc}
\hline & PFI & Latency \\
\hline A Rule & .0559 & $5.9603^{*}$ \\
B Type of Instance & 3.0457 & .1792 \\
C Type of Response & $131.8348^{* *}$ & $12.2256^{* *}$ \\
D Trial Block & $21.4790^{* *}$ & $41.3088^{* *}$ \\
A X B & 1.6817 & $6.1044^{*}$ \\
B X D & .0024 & .4444 \\
A X C & 7.6490 & 1.0820 \\
A X D & $4.8041^{*}$ & .6153 \\
B X C & .0353 & .9517 \\
C X D & 1.8602 & 2.0858 \\
A X B X D & .1083 & .0679 \\
A X X X D & .5844 & .1517 \\
B X C X D & .0459 & 2.2633 \\
\hline
\end{tabular}

$* p<.05, * 0<.01$.

appropriate additions were made to this set of instructions. For example, in the positive-focusing instruction condition, the Ss were also told: "In the past we have found that people do better on these problems if they pay attention primarily to those patterns that are examples of the concept rather than to those patterns which are not examples of the concept." The $\mathbf{S}$ was then required to solve either a conjunctive or a disjunctive problem. For half of the Ss, "red" and "square" were the relevant attributes; the other half of the Ss had "green" and "triangle" as relevant attributes. The $S$ had complete control of stimulus presentation after the first slide was presented.

\section{DEPENDENT VARIABLES}

The total number of trials and errors to criterion was recorded for each $S$. In addition, latencies and S-paced PFIs were recorded. Latency and PFI analyses were performed on the appropriate mean scores for each $\mathrm{S}$. For example, in the comparison of positive and negative instances, individual S's mean latencies and PFIs on positive- and negative-instance trials were compared. Likewise, for the trial block comparison, the mean latencies and PFIs for the first half of the precriterion trials were compared with the mean latencies and PFIs for the second half of the precriterion trials.

\section{RESULTS}

In order to meet the assumption of homogeneity of variance, a logarithmic transformation was performed on the number of both trials and errors to criterion. A 4 (instruction condition) by 2 (rule) by 2 (relevant attributes) analysis of variance was completed on the transformed data. In the analysis of both trials and errors, rule was the only significant source of variance $(\mathrm{F}=50.13$ and 44.73 , $\mathrm{df}=1 / 64, \mathrm{p}<.001$ for trials and errors, respectively). The conjunctive problem was solved more rapidly than the disjunctive problem. The geometric means for the

Table 2

Geometric Means of Latencies and PFIs

Latency

\begin{tabular}{|c|c|c|c|c|c|c|c|c|}
\hline & \multicolumn{4}{|c|}{ Conjunctive } & \multicolumn{4}{|c|}{ Disjunctive } \\
\hline & \multicolumn{2}{|c|}{ Positive } & \multicolumn{2}{|c|}{ Negative } & \multicolumn{2}{|c|}{ Positive } & \multicolumn{2}{|c|}{ Negative } \\
\hline & Right & Wrong & Right & Wrong & Right & Wrong & Right & Wrong \\
\hline Trial 1 & 2.20 & 4.37 & 3.30 & 3.63 & 4.08 & 4.66 & 4.28 & 5.35 \\
\hline \multirow[t]{2}{*}{ Trial 2} & 3.14 & 3.72 & 2.37 & 2.95 & 2.65 & 3.67 & 3.17 & 3.84 \\
\hline & \multicolumn{8}{|c|}{ PFl } \\
\hline Trial 1 & 2.69 & 3.96 & 1.79 & 3.21 & 2.10 & 4.23 & 1.97 & 3.11 \\
\hline Trial 2 & 1.71 & 2.36 & 1.80 & 2.78 & 1.56 & 3.54 & 1.43 & 3.23 \\
\hline
\end{tabular}

conjunctive problem were 11.12 and 1.15 for trials and errors, respectively; the geometric means for the disjunctive problems were 54.93 and 16.77 for trials and errors, respectively.

Since the distributions for the latency and PFI scores were skewed, all analyses were performed on logarithmic transformations of these scores. Table 1 presents the geometric means for both PFI and latency scores. Ideally the data would have been analyses with a mixed analysis of variance. However, since there were so many empty cells (e.g., cases in which the $S$ was never incorrect on a positive instance), the comparisons were made, collapsing across instruction conditions, with individual $F$ tests for both main effects and interactions. Table 2 presents the results of these comparisons.

In the PFI comparisons, trial block and type of response were significant main effects; the PFIs were longer both in the first half of the session and after incorrect responses. The mean PFIs after correct and incorrect responses were 2.22 and $4.32 \mathrm{sec}$, respectively. The interaction between rule and trial block was reliable. Although for both rules the PFIs were longer on the first trial block than on the second, the PFIs for the conjunctive problem were longer than those for the disjunctive problem on the first trial block, while the reverse was true for the second trial block. The interaction between rule and type of response was also significant; although the PFIs were longer after incorrect responses for both rules, the PFIs after correct responses were longer on the conjunctive problem than on the disjunctive problem, while the PFIs after incorrect responses were longer on the disjunctive problem than on the conjunctive problem.

The significant comparisons obtained with the latency data were: (1) rule (latencies were longer on the disjunctive problem); (2) type of response (latencies were longer on the incorrect response trials); (3) trial block (latencies were longer on the first trial block); and (4) the interaction between rule and type of instance. The interaction between rule and type of instance arose because on the conjunctive problem the latencies were longer on positive-instance trials than on negative-instance trials, while the revarse occurred on the disjunctive problem.

Each instruction condition was also analyzed separately, but since these results were generally consistent with those obtained in the combined instruction condition analyses, they will not be reported in detail. The only important finding obtained was that the main effect of rule in the overall latency analysis resulted largely from the significant effect of rule in the Control 1 condition; rule was not significant in the latency analyses of the other instruction conditions.

\section{DISCUSSION}

Neither the main effect of type of instance or the interaction between rule and type of instance were significant in the PFI data. Thus, if the length of the PFI is an index of information processing time as has been proposed (e.g., Bourne, Dodd, Guy, \& Justesen, 1968), it appears that information processing time does not vary as a function of either the type of instance or the complexity of the class of stimuli to be learned.

The most interesting effect in the present study was the PFI difference following correct and incorrect responses. As in the Wells and Laestadius S-controlled condition, the PFIs in the present study were longer after incorrect than after correct responses. These results support the prediction from Levine's theory (Levine, 1966) that Ss should require more time after "wrong" than after "right." Since the PFI on correct response trials was $2.2 \mathrm{sec}$, which is more than the length of time needed to change the stimulus pattern, it also appears that the Ss do some information processing on correct response trials. [The nature of this information processing has not yet been firmly established, but there is some evidence that Ss test hypotheses (e.g., Levine, 1966)]

Since the data from the S-controlled studies indicates that Ss take longer after errors, it is surprising that performance in the E-controlled conditions is not 
facilitated more by long PFIs after errors than by long PFIs after correct responses. As was mentioned previously, Bourne et al found that any increase in PFI, regardless of whether it followed a correct or incorrect response, facilitated performance, while Wells and Laestadius found that long PFIs after correct responses facilitated performance more than long PFIs after incorrect responses. The discrepancy between the E-controlled and S-controlled studies may be a result of the lengths of the PFIs employed. In the S-controlled studies Ss take an average of 2 to $3 \mathrm{sec}$ after correct responses and an average of 4 to $5 \mathrm{sec}$ after errors. If intervals of these lengths were used in an E-controlled study, then the Ss might do better when given the long interval after errors.

In the latency analysis, as in the PFI analysis, the only reliable effects that appear consistently across instruction groups were those of trial block and type of response; latencies were longer during the first half of the session and on error trials. Assuming that during the latency period the $S$ is trying to decide upon the appropriate classification for the stimulus pattern, one possible explanation for the shorter latencies on the correct response trials and during the second half of the session is that the $S$ is more likely to know the correct response to the patterns appearing on these trials and, therefore, does not have to spend time deciding what type of response to make. This explanation is, of course, based on the assumption that some stimulus patterns are learned before others, and there is some evidence which suggests that this is the case (e.g., King, 1966).

\section{REFERENCES}

BOLRNE, L. E., JR., \& BLNDFRSON, C, V. Effects of delay of information feedback and length of postfeedback interval on concept identification. Journal of Experimental Psychology, 1963, 65, 1-5.

BOURNE, L. E., JR., DODD, D. H., GUY, D. E. \& JUSTESEN, D. R. Response-contingent intertrial intervals in concept identification. Journal of Experimental Psychology, 1968, 76, 601-608.

BOURNE, L. E., JR., \& GUY, D. E. Learning conceptual rules. II: The role of positive and negative instances. Joumal of Experimental Psychology, 1968, 77, 488.494.

CONANT, M. B., \& TRABASSO, T. Conjunctive and disjunctive concept formation under equal-information conditions. Journal of Experimental Psychology, 1964, 67, 250-255.

DENNEY, N. W. The effect of varying percentage of positive and negative instances and instructions on conjunctive and disjunctive learning. Psychonomic Science, 1969, 17, 193-194

KING, W. L. A developmental study of rule learning. Joumal of Experimental Child Psychology, 1966, 4, 217-221.

LEVINE, M. Hypothesis behavior by humans during discrimination learning. Joumal of Experimental Psychology, 1966, 71, 331-338.

WELLS, H., \& LAESTADIUS, J. Error-contingent and self-paced post-IF intervals in concept learning. Paper presented at the meeting of the Western Psychological Association, 1968.

\section{A Markovian investigation of English language redundancy}

\author{
KENT B. TAYLOR† \\ The George Washington University, Washington, D.C. 20006
}

Garner, 1962) to terms involved in prediction of letters at various positions in sequences.

The present study is intended to demonstrate a Markovian approach to the analysis of the statistical properties of English language structure and to cite the implications of this new approach in terms of current research in language redundancy. While information-theory measures have been extensively used to identify the redundancy of language, the basic assumptions underlying the use of such measures, namely the qualities of ergodicity and regularity, have not been demonstrated. The information or entropy in nonergodic or nonregular sources is not determinable since statistical equilibrium is never reached.

Among the possible discrete Markov processes there exists a group with special properties of significance in communication theory. This special class of regular ergodic sources has the qualities that some power of the exponentiated matrix has only positive components, and there exists a state, or limit, from which further exponentiations will not change the matrix. Such ergodic sources reach a point of statistical equilibrium at which point the information of the process is defined. A Markovian approach permits validation of the crucial assumptions of ergodicity and regularity and yields additional data about the nature of sequential constraint operating along letter sequences.

Markov processes possess other mathematical properties that enhance their use to the mathematical language theorist. Among these are their ability to determine the probability of being in certain states beyond the succeeding one. Given the transition matrix $P$, and given a starting state $i$, it is possible to determine the probability that after $\mathrm{N}$ steps the process will be in state $j$. This probability is denoted by

$$
P_{\text {ij. }}^{(n)}
$$

To test the assumptions of ergodicity and regularity underlying the application of information-theory measures to language-redundancy studies, digram letter transition probabilities were represented in the form of a Markov source. The source was determined to be ergodic and regular, and sequential letter dependencies were found to be relatively short run. Trigram probabilities predicted by the Markov model were consistently slightly higher than empirical probabilities, demonstrating limitation of such an approach to statistical language analysis.

A direct measurement of the redundancy of printed English is nearly impossible since the number of possible letter sequences is prohibitively large for

*The author expresses his appreciation to the George Washington L niversity Computer Center for the use of its facilitics.

$\div$ Please address reprint requests to 1229 l orest Ave, Palo Alto, Calif. 94301.

Psychon. Sci., 1970, Vol. 20 (4) any direct count. For this reason, redundancy has been investigated through methods of estimating the constraint operating on single letters at the end of long sequences of letters, yielding a measure of average redundancy. The two most widely used methods are Shannon's (1951) "guessing-game" technique, in which the last letter of a sequence is produced from preceding letters, and the multivariate-analysis approach (e.g.,
If one were to view the states of a Markov process defined by digram probabilities as letters of the alphabet and a blank, thus forming a $27 \times 27$ matrix, it would be possible to determine the probability of a given letter a being followed by a letter $b$ after $\mathrm{N}$ transitions or $\mathrm{N}$ subsequent letters. This calculation can be accomplished by raising the transition matrix to the Nth power and reading the probability at the intersection of Row a and Column b. Theoretically, by applying such methods with the square of a $27 \times 27$ digram transition matrix, it should be possible to describe the probabilities of occurrence of trigrams. That this does not occur, 